\title{
Analyte-responsive fluorescent probes with AIE characteristic based on the change of covalent bond
}

\author{
Min $\mathrm{Xu}^{1}$, Xudong Wang ${ }^{2}$, Quan Wang ${ }^{1}$, Qinyu $\mathrm{Hu}^{1}$, Kaixun Huang ${ }^{2}$, Xiaoding Lou ${ }^{1 *}$ and Fan Xia ${ }^{1,2^{*}}$
}

\begin{abstract}
It is important for the determination of biologically and/or environmentally relevant species by utilization of fluorescent probes. Conventional fluorescent probes are subjected to the influence of aggregation-caused quenching that is limiting their application due to low selectivity as well as photobleaching. Additionally, quencher pairs are usually introduced in the design of these probes, which lead to the complex synthetic procedure. A novel class of fluorogens with aggregation-induced emission (AIE) characteristic provide a solution to address the dilemma. By taking advantage of the unique characteristic of AIE fluorogens, specific turn-on probes have been developed via combination with recognition components, exhibiting low background, good selectivity and outstanding photostability. This review focuses on the development of fluorescent probes with AIE characteristics via the bond cleavage as well as formation strategy.
\end{abstract}

Keywords: fluorescent probe, aggregation-induced emission, cleavage, formation, covalent bond

\section{INTRODUCTION}

Over the past decade, fluorescent probes were capable of in situ, non-invasive and real-time monitoring of biological samples and biological processes in cells, tissues and organisms [1-6]. The probes not only give penetrating insights into comprehending physiological changes in basic biology and disease, but also offer a powerful approach for the development of interventional surgical imaging as well as imaging-guided therapies [7-9]. A large number of fluorescent probes $[10,11]$ have been developed on the basis of various fluorescent materials such as quantum dots [12], upconversion luminescence nanoparticles, and organic dyes. Organic dyes are an alternative option which could be employed to serve as a fluorescent probe in terms of biocompatibility and synthesis. The analyte detection is achieved through various reaction mechanisms involving protonationdeprotonation, complexation as well as cleavage and formation of covalent bonds between the fluorescent probe and analyte. Among the reaction mechanisms, the selective detection of analytes on the basis of the cleavage and formation of covalent bonds has attracted much attention. In general, a large majority of fluorescent probes possess excellent selectivity toward analytes compared with these probes through complexation, which provides an alternate approach to detect plentiful analytes with high specificity. Furthermore, most conventional organic dyes exhibit bright fluorescence in dilute solutions. However, they are subjected to fluorescence quenching at high concentrations on account of aggregation-caused quenching (ACQ). In addition, fluorescent probes are utilized at low concentration due to ACQ, leading to the photobleaching of conventional organic dyes.

Recently, another group of fluorescent dyes with aggregation-induced emission (AIE) phenomenon have been reported which was opposite to ACQ phenomenon. In the AIE process, negligible fluorescence is observed at low concentrations, whereas fluorescent dyes display strong fluorescence at high concentrations. A large amount of fluorescent molecules $[13,14]$ with propellershaped structures have been found to display apparent AIE effect. Once they form aggregate, either intramolecular motions (RIM) or intramolecular rotation (RIR) is restricted. The highly twisted molecular conformation is owing to the intermolecular $\pi-\pi$ stacking interaction. Thus, the synergistic effect described above leads to the fluorescence light-up of tetraphenylene (TPE). By utilization of RIR as well as RIM effect, a large

\footnotetext{
${ }^{1}$ Engineering Research Center of Nano-Geomaterials of Ministry of Education, Faculty of Materials Science and Chemistry, China University of Geosciences, Wuhan 430074, China

${ }^{2}$ Hubei Key Laboratory of Bioinorganic Chemistry \& Materia Medica, School of Chemistry and Chemical Engineering, Huazhong University of Science and Technology, Wuhan 430074, China

* Corresponding authors (emails: louxiaoding@cug.edu.cn (Lou X); xiafan@hust.edu.cn or xiafan@cug.edu.cn (Xia F))
} 
number of fluorescence probes [15-19] with AIE characteristics have been developed via various strategies including physical and chemical reactions, i.e., AIE probes based on TPE derivatives $[20,21]$ and 9,10-distyrylanthracene (DSA) derivatives [22-27]. The AIEgens covalently link with the recognition unit to enhance the stability of the probe. Additionally, the probes connect with each other through covalent bond in the presence of target, enabling fluorescence light-up.

In this review, we summarize the AIEgens in the bioapplication on the basis of covalent bond changes of probes in recent years (Scheme 1). The changes of covalent bond include the bond formation as well as the bond cleavage. Once the covalent bond of the probe is cleaved, hydrophobic AIEgens are released. The AIEgens aggregate and subsequently switch-up fluoresces in aqueous media due to either RIM or RIR. Furthermore, when the target is present, the covalent bonds form and thus block the rotation of AIEgens. Finally, this review provides insights into the application of probes based on covalent bond change strategies to life chemistry.

\section{FLUORESCENCT PROBES BASED ON THE CLEAVAGE AND FORMATION OF COVALENT BONDS}

\section{Bond cleavage}

The probes based on the cleavage of covalent bonds by analytes are generally proposed. There are seven types based on the covalent bond cleavage such as amido bond, ester bond, boron ester bond, phosphodiester bond, disulfide bond, $\mathrm{C}-\mathrm{O}$ bond and $\mathrm{S}-\mathrm{O}$ bond. Once the probe is cleaved, the AIEgens form aggregates, leading to fluorescence light-up.

\section{Amido bond}

Direct monitoring of multiple enzyme activities in biological processes is very important for the diagnosis of disease. Liu's group [28] reported a fluorescent probe that can simultaneously monitor the activity of two intracellular caspases. The probe consists of three parts: two (green and red fluorescent) AIE luminophores that can be excited by only single wavelength and a peptide substrate comprising caspase- 8 and caspase- 3 (Fig. 1). The hydrophilic probe of the polypeptide has no fluorescence in the aqueous solution. However, the red and green fluorescence are successively light-up after the polypeptide substrate is hydrolyzed and cleaved by caspase- 8 (between TPETH-DVEDIETD and TPS) and caspase- 3 (between TPETH and DVED), which is activated by hydrogen

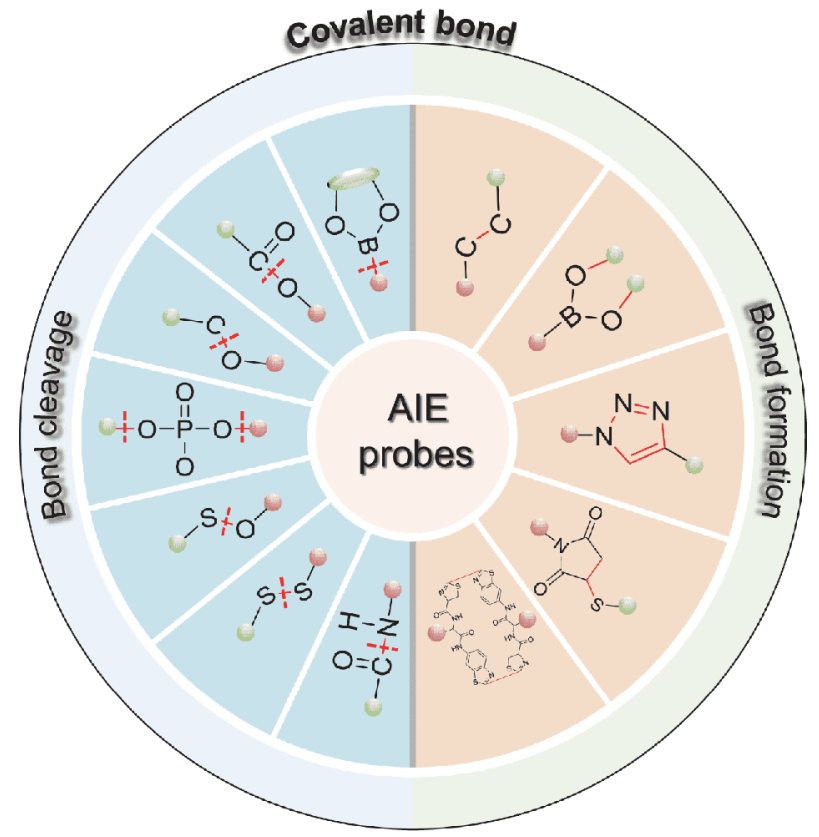

Scheme 1 The strategy of AIE probes based on the cleavage and formation of covalent bond.

peroxide to cause apoptosis in Hela cells. Successively illuminating fluorescence probe can be used to monitor cascade activation during apoptosis, and thus the probe can be used to assess the therapeutic effects of anticancer drugs. This probe is capable in real time and simultaneous detection of multiple enzyme activities in cells during a biological process.

In 2016, our group [29] chemically coupled the broadspectrum anticancer drug doxorubicin (DOX) and the AIE molecule PyTPE to the functional penetrating peptide FCPPs (CRRRRRRRRRPLGLAGPra- $\mathrm{NH}_{2}$ ) via the "maleimide-thiol" click reaction and the "azido-alkynyl" click reaction. The probe DOX-FCPPs-PyTPE (DFP, Fig. 2) was synthesized with the response to matrix metalloproteinase-2 (MMP-2) for integration of diagnosis and treatment. DFP can be specifically cleaved (between LG and LAG) in the presence of MMP-2 to form a therapeutic moiety DOX-MHS-CRRRRRRRRRPL containing a penetrating peptide fragment and an imaging moiety LAGPra-PyTPE containing a hydrophobic fragment. The therapeutic molecules originally binding with the membrane-penetrating peptide are separated from the imaging molecule. The transmembrane peptide binds only to DOX alone. DOX can be transported faster into the cell by the penetrating peptide and kill the cancer cells. The PyTPE molecule originally binding to the hydrophilic transmembrane peptide is dispersed. After se- 


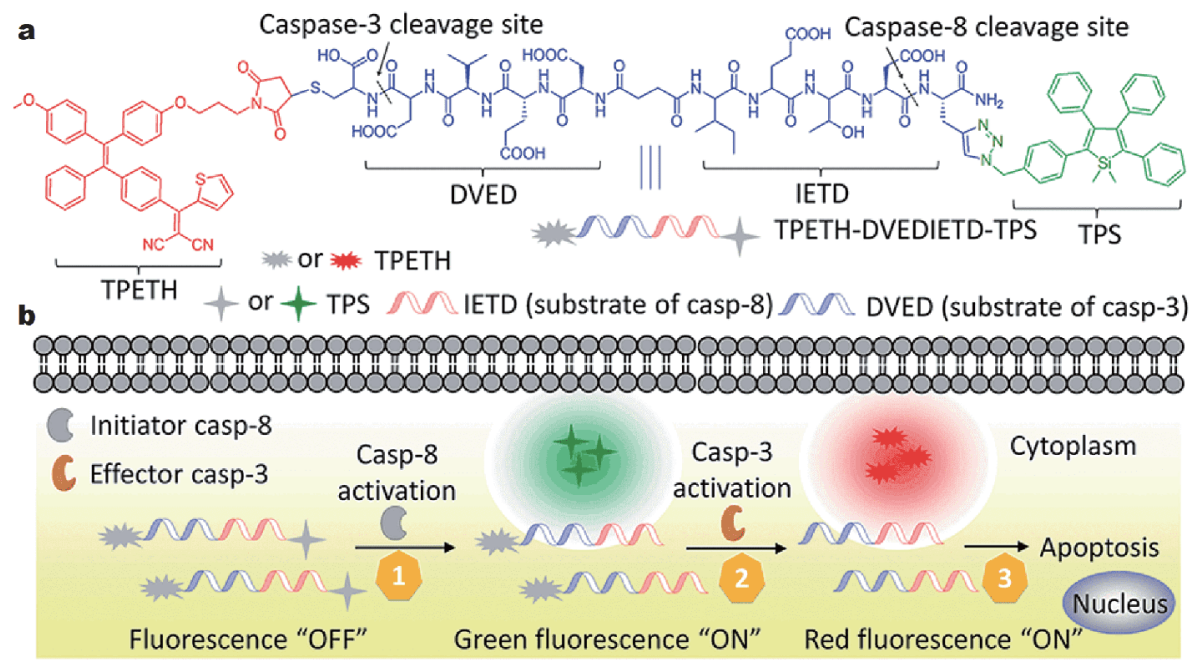

Figure 1 Chemical structure (a) of a single fluorescence probe for real-time imaging (b) of the caspase activation.

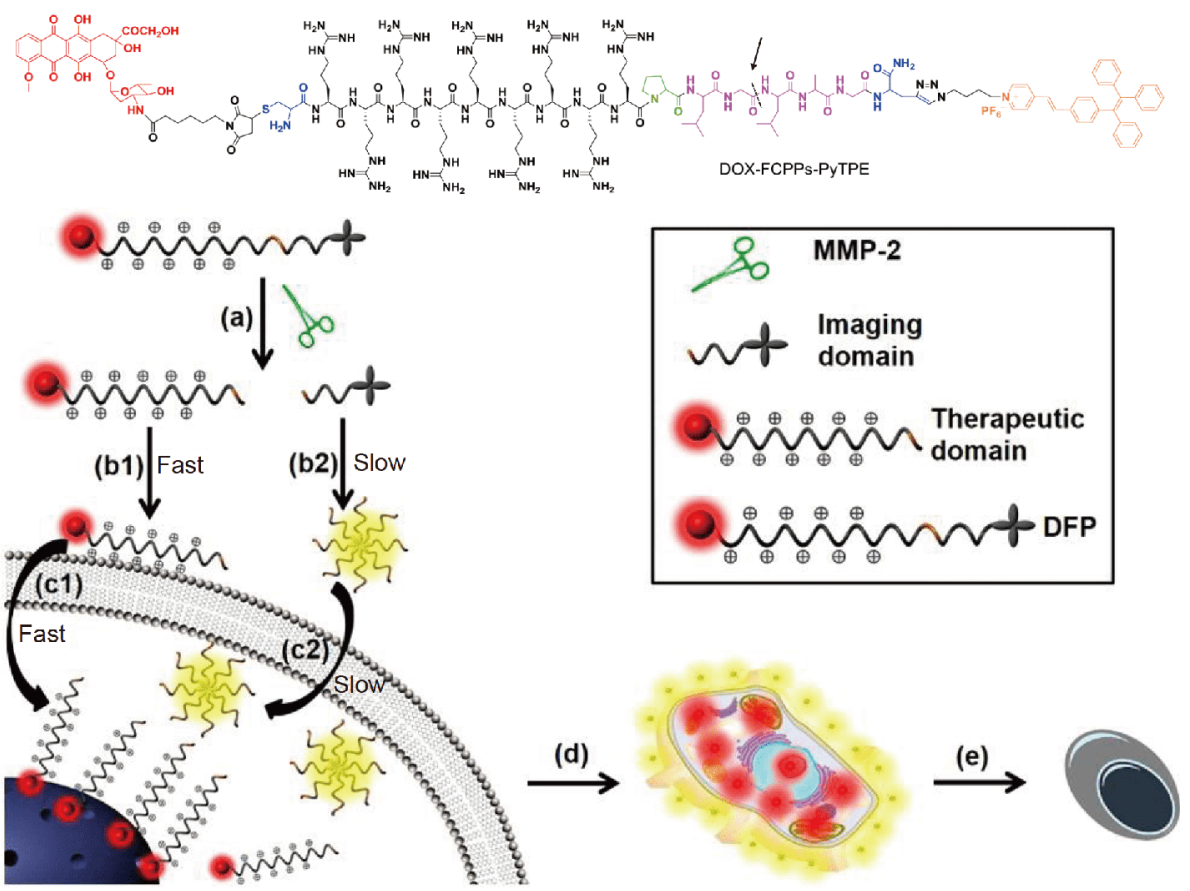

Figure 2 The detection mechanism of the probe DFP for drug delivery and release tracking in specific cancer cells. Reproduced with permission from Ref. [29]. Copyright 2016, American Chemical Society.

paration from the transmembrane peptide, the hydrophobicity of PyTPE is enhanced so as to aggregate and emit yellow fluorescence. Therefore, DFP can not only control the drug release, but also efficiently kill the cell lines with high expression of MMP-2, simultaneously tracking the release of the drug in real time according to the changes of the two kinds of fluorescence, so as to achieve the purpose of integration of diagnosis and treatment.

The activatable photosensitizers are widely used in fluorescence real-time imaging and photodynamic therapy. Nevertheless, the conventional photosensitizers tend to aggregate in water resulting in a decrease in fluorescence quenching and photodynamic effects. Based on the 


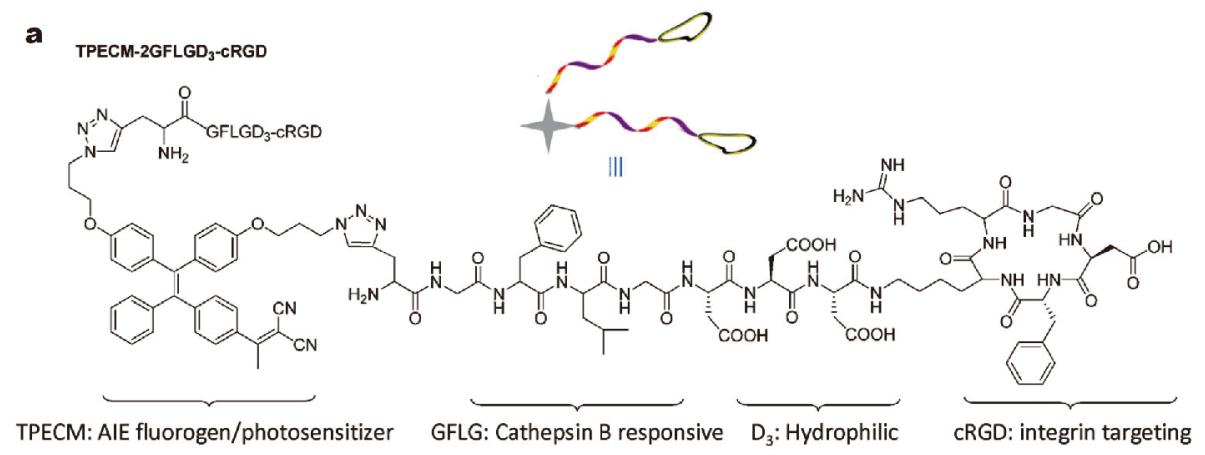

b

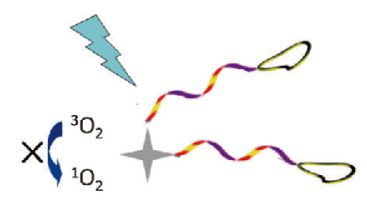

Fluorescence "off"
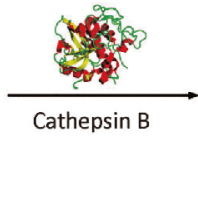

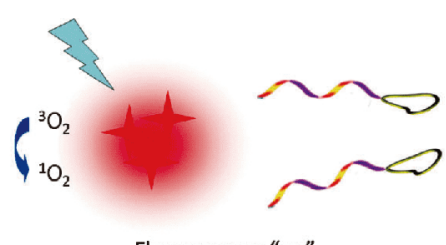

Fluorescence "on"

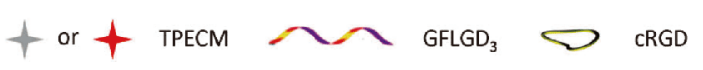

Figure 3 (a) Schematic of the probe TPECM-2GFLGD3-cRGD. (b) The mechanism of probe activation by cathepsin B and irradiated to generate reactive oxygen species (ROS). Reproduced with permission from Ref. [30]. Copyright 2015, Wiley.

recently developed AIE fluorophore, Liu's group [30] designed a simple and unique probe for turn-on targeted fluorescence imaging and activatable photodynamic therapy. As presented in Fig. 3, this probe consists of four parts: 1) in which the yellow fluorescent AIE luminophore acts as a fluorescent imaging unit as well as a photosensitizer, 2) the GFLG peptide is a substrate for cathepsin B, 3) a hydrophilic linker with three Asp (D) can improve the water solubility of the probe, 4) cRGD serves as a targeting moiety. The probe has almost no fluorescence in aqueous solution due to good dispersibility and the limited ability to produce singlet oxygen. After the probe is taken up by the cancer cells, the cleavage of the probe between TPECM-GFLG and $\mathrm{D}_{3}$ cRGD occurrs by the hydrolysis of cathepsin $\mathrm{B}$ and the GFLG substrate is released. The released probe aggregates due to enhanced hydrophobicity resulting in an increase in fluorescence signal accompanied by activation of probe photodynamic therapy. Therefore, this probe provides an efficient way to design activatable photosensitizers without quenching groups or energy acceptors.

A variety of AIE-functionalized probes based on enzyme-actived cleavage reactions have been developed via a general assay, and these fluorescent turn-on bioprobes can be used for monitoring matrix metallopeptidase 13 [31], dipeptidyl peptidase-4 [32], $\gamma$-glutamyl transpeptidase [33], chymase [34], angiotensin converting enzyme
[35], caspase-3 [36-39], real-time imaging cancer cell apoptosis [40-44], differentiation in living stem cells [45] and targeted drug delivery and therapeutic [46].

\section{Ester bond}

Lipase in blood serum is a crucial indicator of acute pancreatitis. It is urgent to develop a stable and efficient probe for sensing lipase levels. Tang et al. [47] constructed a novel light-up AIE bioprobe S1 for sensing the lipase. It is well known that lipase is capable of achieving optimal catalytic performance at the oil-water interface and is an enzyme for heterogeneous catalysis. The hydrophobicity of the AIE probe S1 is improved because two glutamate groups are functionalized onto the TPE. And the hydrophilicity would increase via the existence of amino and carboxyl parts, in favor of contacting with lipase in the water media at the oil-water interface. These hydrophilic groups generate an interfacial control effect. As illustrated in Fig. 4, the ester group will cleave while treated with lipase and the probe S1 will turn to 4,40dihydroxy tetraphenylethylene (TPE-2OH) which aggregates in the solvent, leading to strong blue emission. Since the glutamate group is a specific substrate for the lipase hydrolysis reaction, the $S 1$ probe possesses superior selectivity. And because of the interface control effect, S1 can detect some real biological samples with low lipase content, such as blood serum. It is the first report on the 


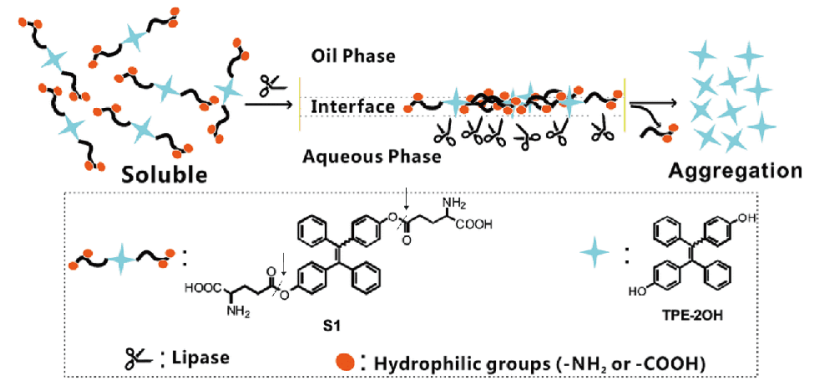

Figure 4 The detection strategy and chemical structure of probe S1. Reproduced with permission from Ref. [47]. Copyright 2017, the Royal Society of Chemistry.

detection of lipase in real human serum samples by fluorescent probes with the shortcomings of high detection and low sensitivity of lipase probes, and establishes an analysis method for early warning of acute pancreatitis. This study of detecting lipase in actual samples not only broadens the range of AIE-based enzyme assays, but also provides a new way to increase the efficiency of interfacial catalysis.

In their subsequent work [48], a patent fluorescent light-up bioprobe was constructed with a limit of detection of $0.1 \mathrm{mg} \mathrm{mL}^{-1}$ for lipase activity based on TPE derivative P1 with an ester group which can be activated. And Liu's group [49] utilized a cleavable aminoacrylate linker which responded to singlet oxygen to conjugate a fluorescent green emissive rhodol dye and a red AIEbased photosensitizer to realize real-time monitoring of ROS generation during photodynamic therapy.

\section{Boron ester bond}

In 2016, Xing's group [50] designed and synthesized a novel AIE molecule, tetraphenylethene derivative (TPEDABA) which can specially respond to $\mathrm{H}_{2} \mathrm{O}_{2}$ when connected with D-fructose (TPE-DABF). For the AIE characteristic, the probe has low background, and when treated with hydrogen peroxide, the boronic ester cleaves from the TPE-DABF and the TPE shows a bathochromic shift. TPE-DABF can be used as an intramolecular charge transfer (ICT) probe because there are electron-withdrawing group (aldehyde) and electron-donating group (phenoic hydroxyl) in the oxidation product TPE-DAP. Remarkably, as depicted in Fig. 5, TPE-DABF can also detect $\mathrm{H}_{2} \mathrm{O}_{2}$ generated from the glucose oxidase catalyzed oxidation reactions. And it shows potential applications in detecting $\mathrm{H}_{2} \mathrm{O}_{2}$ generated from different oxidation reactions and facilitates the development of other ROS detectors based on AIEgens.

In addition, Ding et al. [51] proposed an AIE-active turn-on nanoprobe on the basis of TPE derivative, TPEIPB, whose phenylboronate acted as a reaction site for $\mathrm{ONOO}^{-}$. The nanoprobe is able to detect $\mathrm{ONOO}^{-}$within milliseconds and the boronates can effectively compete with self-decomposition and other reactions. Thus the turn-on nanoprobe can selectively image inflammation region and efficiently visualize the treatment efficacy of anti-inflammatory agents in vivo.

\section{Phosphodiester bond}

Enzyme-assisted detection of microRNAs has highly

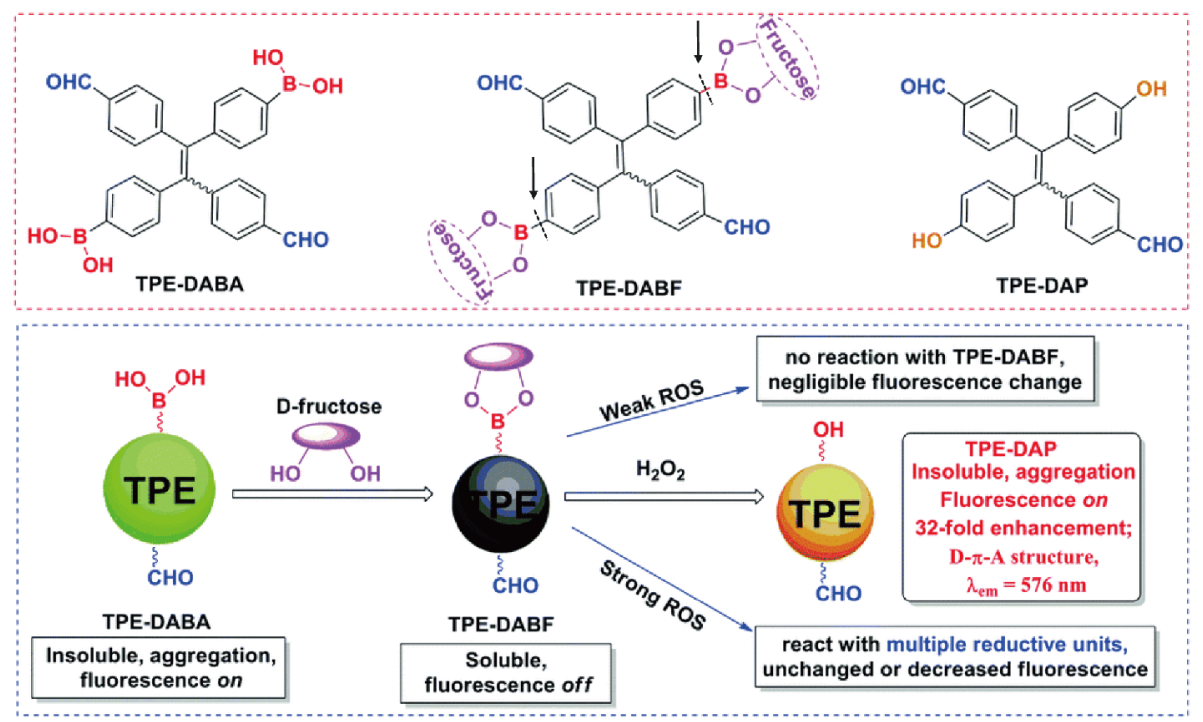

Figure 5 Schematic of AIEgens for determination of $\mathrm{H}_{2} \mathrm{O}_{2}$. Reproduced with permission from Ref. [50]. Copyright 2016, the Royal Society of Chemistry. 
sensitivity as well as specificity in vitro. But the detection of microRNAs in vivo is still a significant challenge due to the low content of microRNAs in vivo as well as the complexity of the environment. Based on our previous work [52], we developed a nucleic acid probe (TPEPyLDNA) without a quenching group by click reaction via using a photostable AIE fluorophore [53]. A hydrophobic yellow fluorescent group with AIE characteristics serves as a light-emitting unit, and a hydrophilic nucleic acid 5'CAGTCTGATAAGCTA-3' acts as a recognition unit which hybridizes with microRNA to form a double strand. The probe TPEPy-LDNA in the formed double strand can be hydrolyzed by exonuclease III to release the hydrophobic TPEPy, and thus emits fluorescence. TPEPy-LDNA can be used to detect microRNAs in urine as well as in cells. Because of the high sensitivity of TPEPy-LDNA, it can be used to distinguish the urine of cancer patients from that of normal. In addition, the fluorescence intensity of the probe in MCF-7 cells after $60 \mathrm{~min}$ is higher than that in HeLa cells and HLF cells, indicating that the fluorescent probe exhibits high photostability. As a control, the other two probes containing FAM and $\mathrm{Cy} 3$ with quenching groups are affected by photobleaching. As a consequence, the control group probes are not suitable for long-term monitoring of intracellular microRNAs (Fig. 6). Therefore, these AIE fluorophore-based probes are expected to be useful for detecting cancer markers.

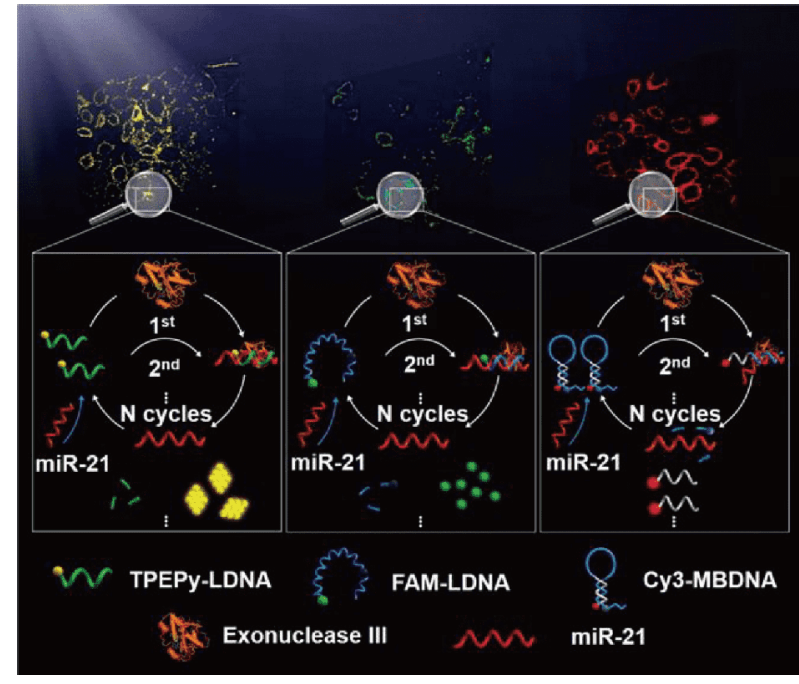

Figure 6 Schematic of in vivo miRNA detection by TPEPy-LDNA. Reproduced with permission from Ref. [53]. Copyright 2016, American Chemical Society.

The detection of low concentrations of mRNA is important in gene-related prognostic analysis. Our group [54] developed a nucleic acid probe (TPE-R-DNA) that combined Exo III target cyclic signal amplification strategy and DNA covalently linked TPE derivatives for tumor tissue imaging and prognosis analysis. The TPE-RDNA consists of two parts: a hydrophobic TPE derivative as a long-wavelength fluorescent imaging moiety and a
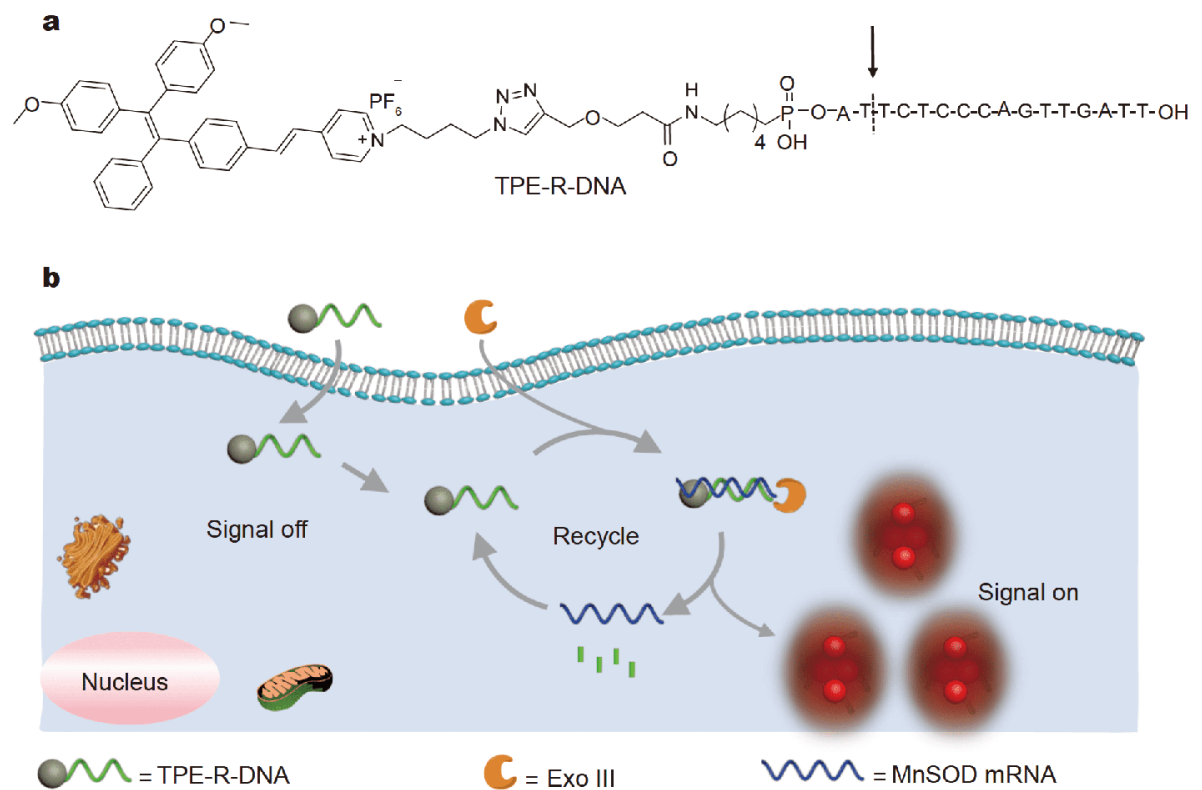

Figure 7 (a) Chemical structure of TPE-R-DNA and (b) the detection mechanism for MnSOD mRNA based on the TPE-R-DNA probe. Reproduced with permission from Ref. [54]. Copyright 2018, American Chemical Society. 
hydrophilic DNA (Alk-DNA) as a target recognition moiety (Fig. 7). In the absence of a target, there is almost no fluorescence in water due to the good dispersibility of TPE-R-DNA in aqueous solution. When the target is present, the TPE-R-DNA hybridizes with the target mRNA to form a double strand, and the probe TPE-RDNA in the formed double strand can be hydrolyzed by Exo III, subsequently the target as well as the hydrophobic TPE-R-AT are released. The released target binds to another probe for the next cycle. While the hydrophobic TPE-R-AT aggregates in the aqueous solution, a gradual increase in fluorescence can be observed. For the first time, the product after hydrolysis is confirmed by mass spectrometry and high-performance liquid phase which contains two bases of $\mathrm{A}$ and $\mathrm{T}$. In addition, the detection limit for detecting target mRNA is as low as $0.6 \mathrm{pmol} \mathrm{L}^{-1}$. TPE-R-DNA can also detect the amount of mRNA in tissues. The results show that the content of MnSOD mRNA in renal cell carcinoma is lower than that in adjacent tissues. The amount of mRNA expression in tissues can be used for prognostic analysis for of cancer patients. In summary, patients with lower MnSOD mRNA levels have a shorter survival period. Therefore, TPE-R-DNA probes have potential for prognostic analysis of disease.

Our group had proposed varieties of facile and sensitive AIEgens modified nucleic acids probes. For example, the probe TPE-Py-DNA [55] can be a superior sensor for telomerase activity with the help of exonuclease III and the probe AFNAs [56] can detect $\mathrm{Hg}^{2+}$ ions in cells and actual samples with duplex-specific nuclease enzyme induced isothermal amplification.

\section{$\mathrm{C}-\mathrm{O}$ bond}

a-Amylase is critical for the carbohydrate digestive system in the human body, which has been continually explored as a crucial indicator of some diseases such as psychological stress. Nevertheless, presently the existing methods including the detection kit, suffer from time consumption, low sensibility indirect assay with the aid of tool enzyme or inhibitor of competitive substrates. Therefore, they are inappropriate for slight and nondisruptive detection of $\alpha$-amylase in body fluids. A facile, intuitive, and sensitive $\alpha$-amylase detection in body fluids remains difficult and challenging. Tang et al. [57] designed a facile fluorescence method based on AIEgens small molecules for the determination of $\alpha$-amylase activity with favorable sensibility. This method provides a simple technique for clinical diagnosis of related diseases due to its high specificity. As shown in Fig. 8, the S2

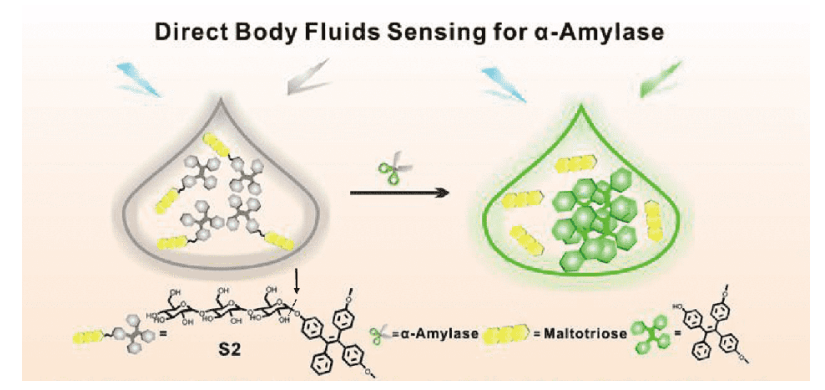

Figure 8 The detection mechanism of probe S2 in a-amylase activity sensing. Reproduced with permission from Ref. [57]. Copyright 2018, American Chemical Society.

probe is composed of a TPE substituted by two methoxy groups as core which connects with a maltotriose unit. The probe is soluble in water due to the oligomeric glucose. And the S2 probe possesses AIE characteristic which emits a negligible fluorescence in water. Upon the addition of $\alpha$-amylase, $\mathrm{C}-\mathrm{O}$ bonds in the probe cleave, and the maltotriose part is given off, remaining TPE aggregating in water media. Consequently, fluorescence turns on. Therefore, a facile fluorescent light-up platform for detecting $\alpha$-amylase can be constructed with S2; moreover, the probe possesses high selectivity due to the specific enzymatic reaction and its AIE characteristic overcomes the shortcomings of traditional assays for $\alpha$-amylase. To our knowledge, this organic small molecule fluorescence sensing system for $a$-amylase is firstly reported. Moreover, they prove its applications in the determination of $\alpha$-amylase activities with rapidity and high sensitivity in patients' body fluids.

Similarly, there is a nanoprobe [58] based on TPE derivative (TPE-3) which changes into TPE-2 due to the cleavage of $\mathrm{C}-\mathrm{O}$ bond in the absence of hydrogen sulfide. So the nanoprobe can be used for imaging hydrogen sulfide in zebrafish.

\section{$S-O$ bond}

In photodynamic therapy, the existing photosensitizers can easily kill normal cells due to their inherent phototoxicity and lack of targeting to cancer cells, which is undesirable. Therefore, there is an urgent need for an activatable photosensitizer that has superior therapeutic efficiency for cancer cells but minimizes side effects on normal cells. On the basis of a newly synthesized AIEgen photosensitizer, Liu et al. [59] developed a glutathione (GSH)-activated and dual-targeted probe for image-guided photodynamic therapy with superior selectivity. The probe exhibits almost none fluorescence or ROS generation ability because of the thiol cleavable quencher group 


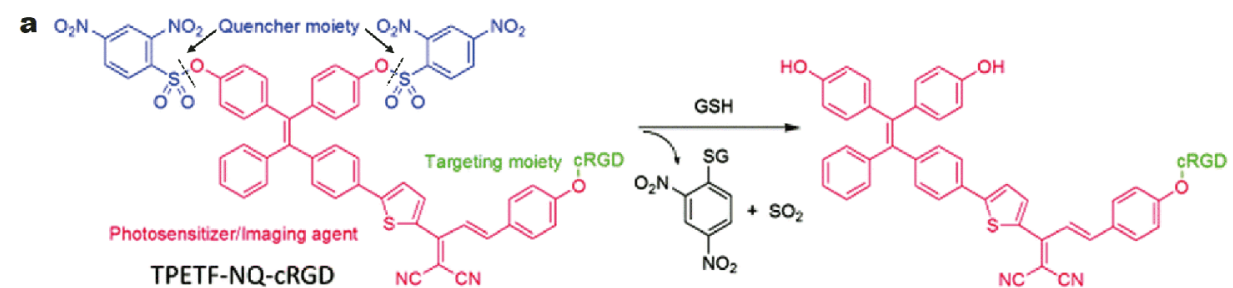

b

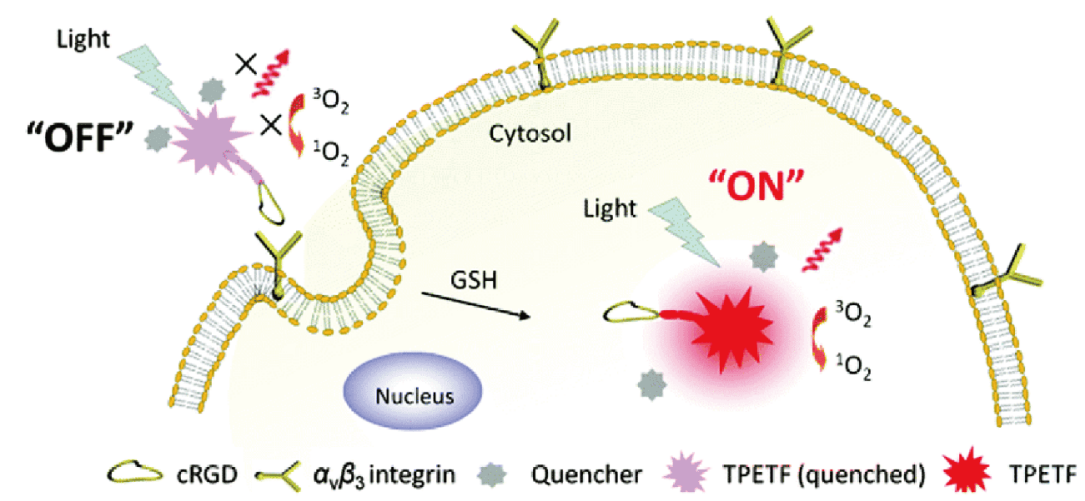

Figure 9 (a) The mechanism of probe TPETF-NQ-cRGD activated by GSH. (b) Schematic of the GSH-activated probe for imaging and ablation of specific cancer cells. Reproduced with permission from Ref. [59]. Copyright 2016, the Royal Society of Chemistry.

2,4-dinitrobenzenesulfonyl. As depicted in Fig. 9, the photosensitizer activity is restored and the fluorescence is light-up after receptor such as $\alpha_{v} \beta_{3}$ integrin mediated endocytosis (1st target). Following activation, the quencher group 2,4-dinitrobenzenesulfonyl is cleaved by intracellular GSH (2nd target). Compared with minimized side effects on normal cells such as NIH 3T3 and $293 \mathrm{~T}$ cells, there is a superior selective imaging and ablation of MDA-MB-231 cancer cells which overexpress $\alpha_{v} \beta_{3}$ integrin due to the high-efficiency ROS ability of the aggregated AIEgen photosensitizer and the double targeting system. Moreover, this dual-target probe offers a higher target-to-background ratio than the available systems before. This AIEgen photosensitizer based GSHactivated probe can be used for cancer therapy. On the basis of this assay concept, it is expected to develop more facile and specific cancer cell targeting elements to apply in practical theranostic in vivo.

\section{Disulfide bond}

Liu et al. [60] developed an integrin $\alpha_{v} \beta_{3}$ targeted GSHresponsive turn-on fluorescent AIE bioprobe for intracellular thiol imaging. Due to the unique characteristic of the AIE molecule, the probe is almost non-fluorescent in aqueous solution, but emits intense fluorescence upon cleavage of the disulfide bond by the thiol. As shown in Fig. 10, the cleavage of the disulfide bond by the thiol results in enhanced fluorescence signal output. The probe allows for real-time fluorescence feedback monitoring of free thiols in solution and in cells with high signal-tonoise ratios. The cRGD-functionalized peptides enable selectively targeting $\alpha_{v} \beta_{3}$ integrins of many angiogenic cancer cells such as U87-MG cells, creating new opportunities for specific intracellular thiol imaging. This method can be used for various applications by just changing the disulfide bond to other cleavable linker groups. Thus, the design of such covalently bound AIE probes has facilitated the development of various selective targeting probes for applications such as imaging, diagnostics and drug screening.

\section{Bond formation}

The probes with AIE feature are designed on the basis of the formation of covalent bonds during chemical reaction. This strategy makes use of the nucleophilic addition reactions between functionalized AIEgens and analytes or click reaction between azide and alkyne or oligomerization of the probes in the presence of analytes. Upon covalent bonds formation, the fluorescence switches on owing to RIR as well as RIM effect in the presence of analytes.

\section{$C-C$ bond}

Since inflammatory diseases increase the risk of cancer, 

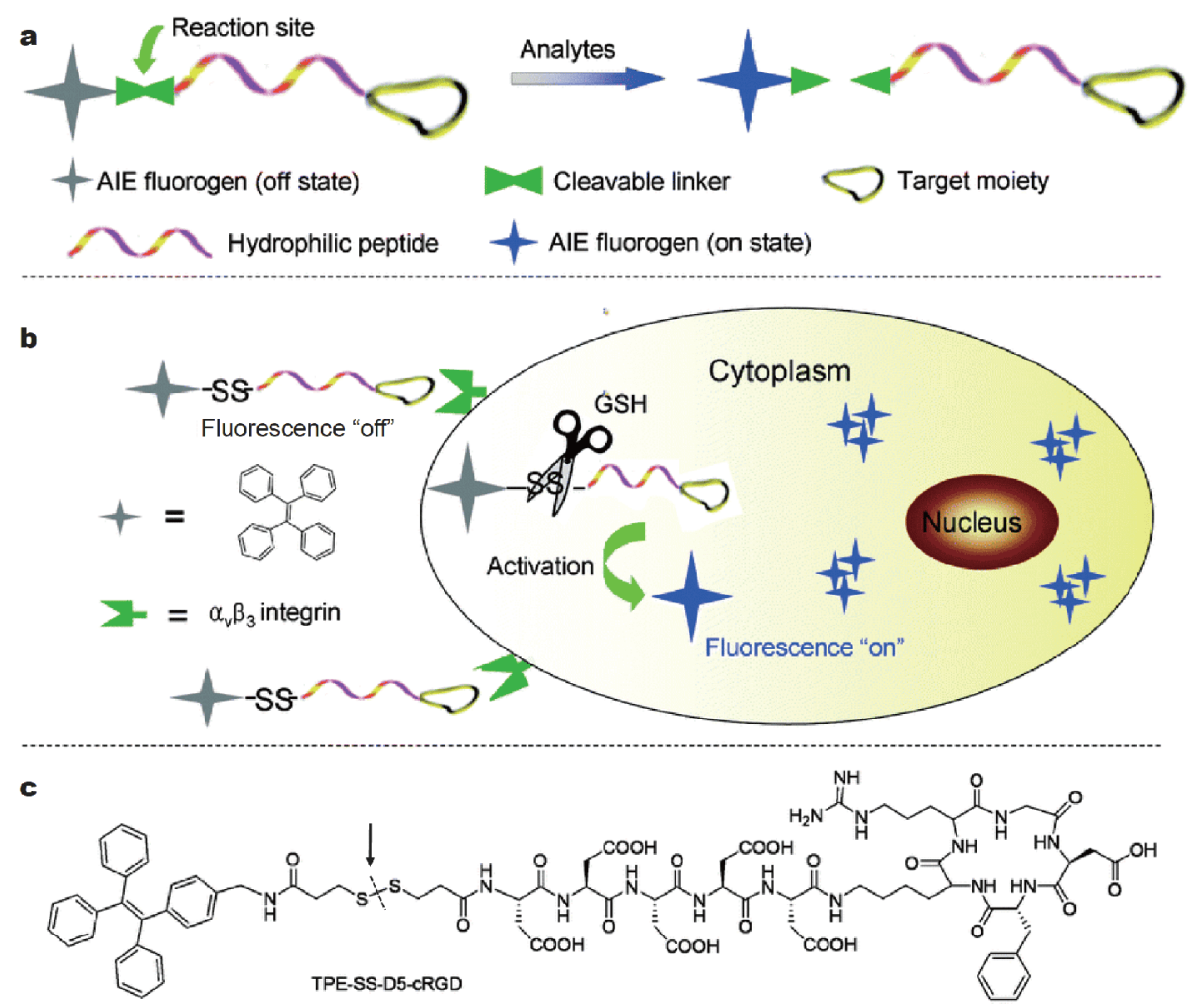

Figure 10 (a) Schematic design of the probe TPE-SS-D5-cRGD. (b) The detection mechanism for imaging of intracellular thiols. (c) The chemical structure of the bioprobe. Reproduced with permission from Ref. [60]. Copyright 2014, the Royal Society of Chemistry.

inflammatory cells have attracted extensive attention. Therefore, the detection and treatment of inflammatory cells is a top priority. We designed a probe combining tyrosine covalently linked tetraphenyl ethylene derivatives (TT) [61], in which the middle tetraphenylethylene acted as the luminescent unit and the tyrosine linked on both sides served as the ROS response unit (Fig. 11). TT is capable of self-assembly by $\mathrm{H}_{2} \mathrm{O}_{2}$ and myeloperoxidase to form long chains which are useful for selective imaging and inhibition of inflammatory cells. Since a hydrophilic amino acid is introduced onto the tetraphenylethylene, the hydrophobic TPE becomes a hydrophilic TT so that there is almost no fluorescence in the aqueous solution. When $\mathrm{H}_{2} \mathrm{O}_{2}$ and myeloperoxidase are present, the tyrosine attaching to both sides of the TT can form aggregates by $\mathrm{C}-\mathrm{C}$ coupling, thereby activating the fluorescence of TPE. The change in molecular orbital amplitude energy after the reaction is further confirmed by density functional theory (DFT) calculation. Thus, based on the enhanced results of TT fluorescence, TT can also be used to selectively image intracellular $\mathrm{H}_{2} \mathrm{O}_{2}$ and myeloperoxidase overexpression. Finally, aggregates of TT cause damage to intracellular mitochondria and apoptosis. This is the first report about the imaging and inhibition of inflammatory cells in response to $\mathrm{H}_{2} \mathrm{O}_{2}$ and myeloperoxidase on the basis of AIEgens.

Besides, Liu's group [62] modified TPE with four tyrosines, TPE-Tyr which dissolved well in water phase with weak emission. The tyrosine portions in TPE-Tyr crosslink covalently with strong emission via enzymatic catalysis so as to sense $\mathrm{H}_{2} \mathrm{O}_{2}$ and glucose. Ding et al. [63] developed a smart organic function-transformable nanoparticle (NP) DTE-TPECM. The NP is composed of a DTE core and two surrounding TPECM units which can reversibly transform between closed-ring and open-ring isomers via light irradiation accompanying with $\mathrm{C}-\mathrm{C}$ bond changes, realizing closed-ring for photoacoustic imaging, and open-ring for fluorescence imaging and photodynamic therapy.

\section{Boron ester bond}

The artificial stimulation-responsive nanopore mimics the gating phenomenon in the organism that allows ions to pass through the cell membrane channel, causing widespread concern. Nevertheless, many factors in the reaction system affect the ion current. Our group [64] 


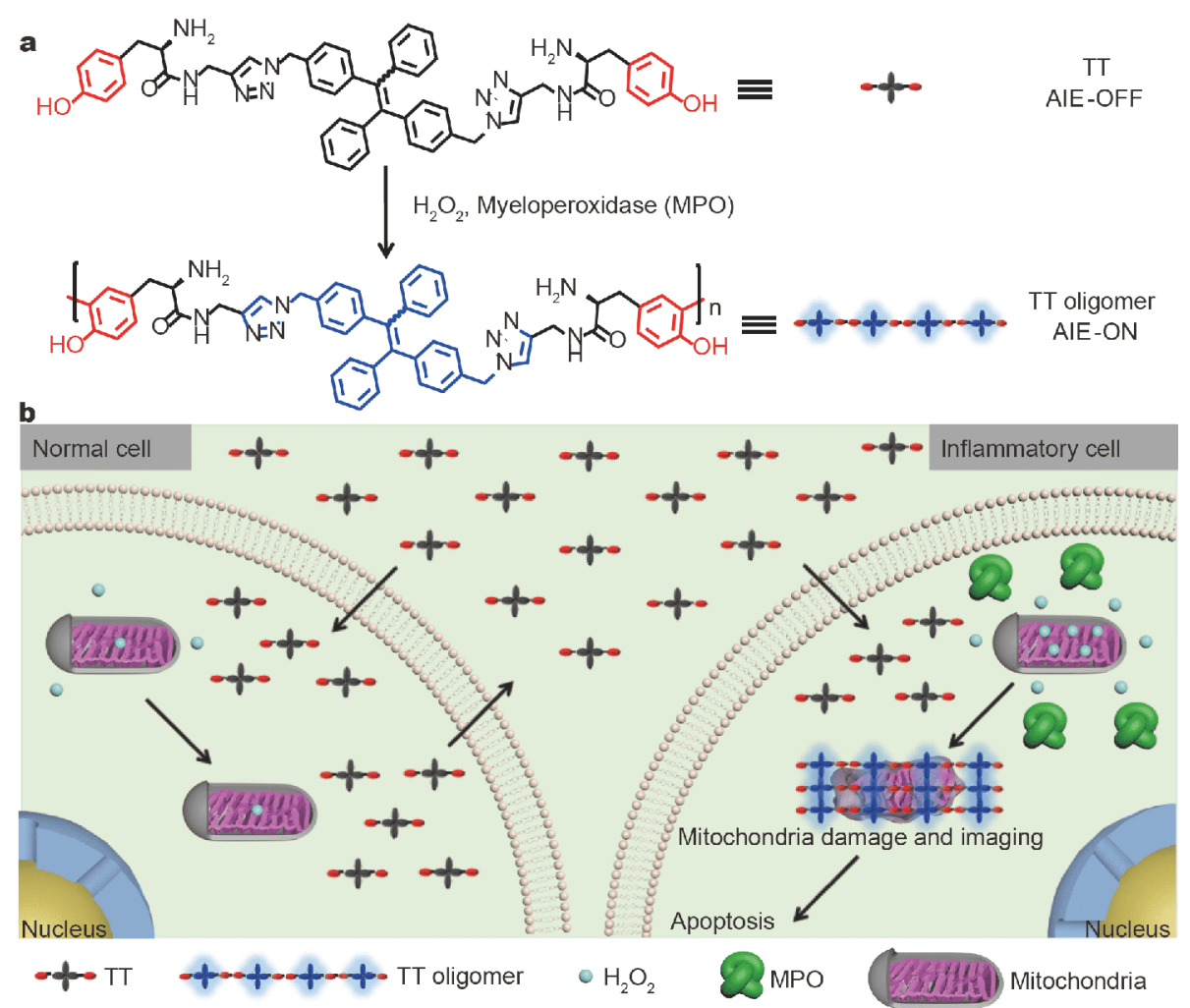

Figure 11 (a) Schematic of $\mathrm{H}_{2} \mathrm{O}_{2}$ detection based on TT. (b) The selective detection and imaging mechanism of TT in co-cultured cells. Reproduced with permission from Ref. [61]. Copyright 2018, Wiley.

demonstrated the gating phenomenon of nanopore by using the fluorescence and ionic current dual signal output of the nanopore (Fig. 12). In addition, the opening and closing of the nanopore is confirmed by analyzing the fluorescence and ionic current of the nanopore, and the results obtained are consistent with the results of the molecular dynamics simulation. When glucose is present, the reduction in ionic current and the enhancement of fluorescence can be immediately observed by the polymerization between the AIE molecule (TPEDB) and the formation of the boric acid diester between the glucose. This method has high sensitivity and selectivity for glucose. In order to verify the feasibility of this method for the clinical application, the glucose contents of 40 urine samples including 10 normal people and 10 diabetic patients before and after treatment were detected. The above results are consistent with the results of the standard curve used in hospitals, which further demonstrates the feasibility of this method. In addition, the dual-signal output nanopore has good resistance to interference from hydrogen peroxide and ascorbic acid in the real environment. Based on the aggregation between AIE mo- lecules, it provides an effective way to develop intelligent gating. Changes from single to dual signals can enable nanopores to be used in complex environments for diagnostics, drug detection, and the study of biomolecule transport processes.

In addition, Tang et al. [65] proposed a turn-on sensor for D-glucose based on the boron ester bond formation among the AIEgens and D-glucose leading to the emission.

\section{Thioester bond}

Although many methods are applied for detecting acetylcholinesterase (AChE) activity and organophosphorus pesticides (OPs), the practical application of most methods is limited by their inherent shortcomings, such as complex analysis processes, long analysis times, and high costs. Therefore, to develop a facile, rapid and sensitive method for detecting AChE activity and OPs is urgent. Li's group [66] constructed a paper-based fluorescent sensor (PFS) with a new AIE fluorogen for facile, efficient, robust and convenient determination of AChE activity and OPs. Maleimide group functionalized tetra- 


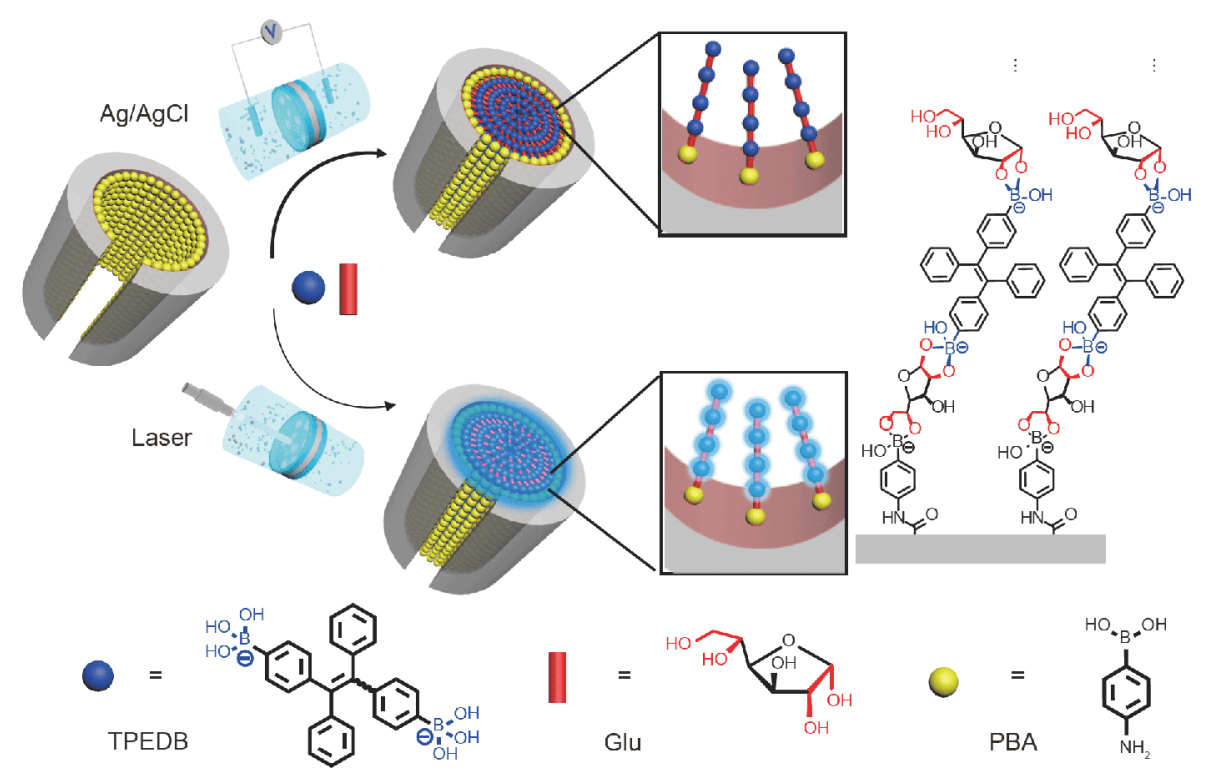

Figure 12 Illustration of gating phenomenon based on the fluorescence and ionic current dual signal output of the nanopore. Reproduced with permission from Ref. [64]. Copyright 2016, Springer Nature.

phenylethene ramification, TPE-M, has almost no fluorescence because of the exciton annihilation process between the carbonyl $(\mathrm{C}=\mathrm{O})$ and the olefinic $(\mathrm{C}=\mathrm{C})$ groups in its maleimide unit. The maleimide of TPE-M cleaves in the presence of thiol choline and changes into TPE-M-S with strong emission while treated with the hydrolysis product of acetylthiocholine (ATCh) catalyzed by AChE. Therefore, by dropping $1 \mu \mathrm{L}$ of the TPE-M solution and $1 \mu \mathrm{L}$ ATCh solution on the test paper, the PFS would realize. To determine AChE activity, the mixture solution reacts with AChE and forms TPE-M-S on PFS with a strong emission which could be visible to the naked eye under UV irradiation. However, AChE activity is inhibited upon the addition of OPs, leading to the reduction of the fluorescence of PFS. And this strategy allows for the convenient, sensitive and visual determinations of OPs in real samples. Without the complex process, fluorescence quenching effects and other issues, this sensor can easily and conveniently visualize AChE activity and OPs in a rapid detection process of only about $180 \mathrm{sec}$. Therefore, the PFS shows great potential for detecting AChE activity and OPs in practical applications.

\section{Nitrogen heterocycle}

Since bio-orthogonal tumor labeling is covalently linked, it could deliver imaging agents or drugs to tumor sites more efficiently than activatable targeting strategies. However, because of the lack of turn-on probes for bio- orthogonal labeling, tumor targeting imaging via bioorthogonal labeling currently depends on elimination reactions to distinguish targets from the original signal of probe. Liu and her colleagues [67] reported for the first time that the fluorophores based on the AIE effect were used for in vivo turn-on bio-orthogonal labeling. As shown in Fig. 13, the BCN-TPET-TEG probe consists of the AIE photosensitizer (TPET), hydrophilic triethylene glycol (TEG) and bicyclo[6.1.0]decyne (BCN) for copperfree click reactions. Among them, hydrophilic TEG imparts good dispersibility of BCN-TPET-TEG in aqueous solution. Therefore, the probe has a weak fluorescence in aqueous solution, which makes the background relatively

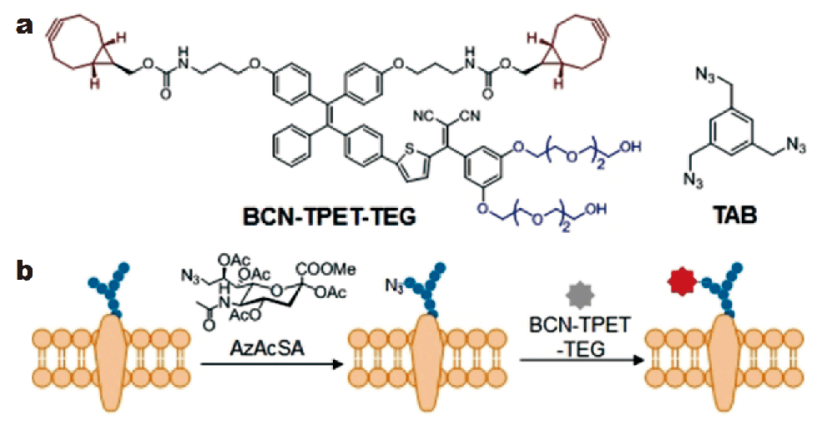

Figure 13 (a) The chemical structure of BCN-TPET-TEG. (b) Schematic of light-up bio-orthogonal tumor labeling based on BCN-TPETTEG. Reproduced with permission from Ref. [67]. Copyright 2018, Wiley. 


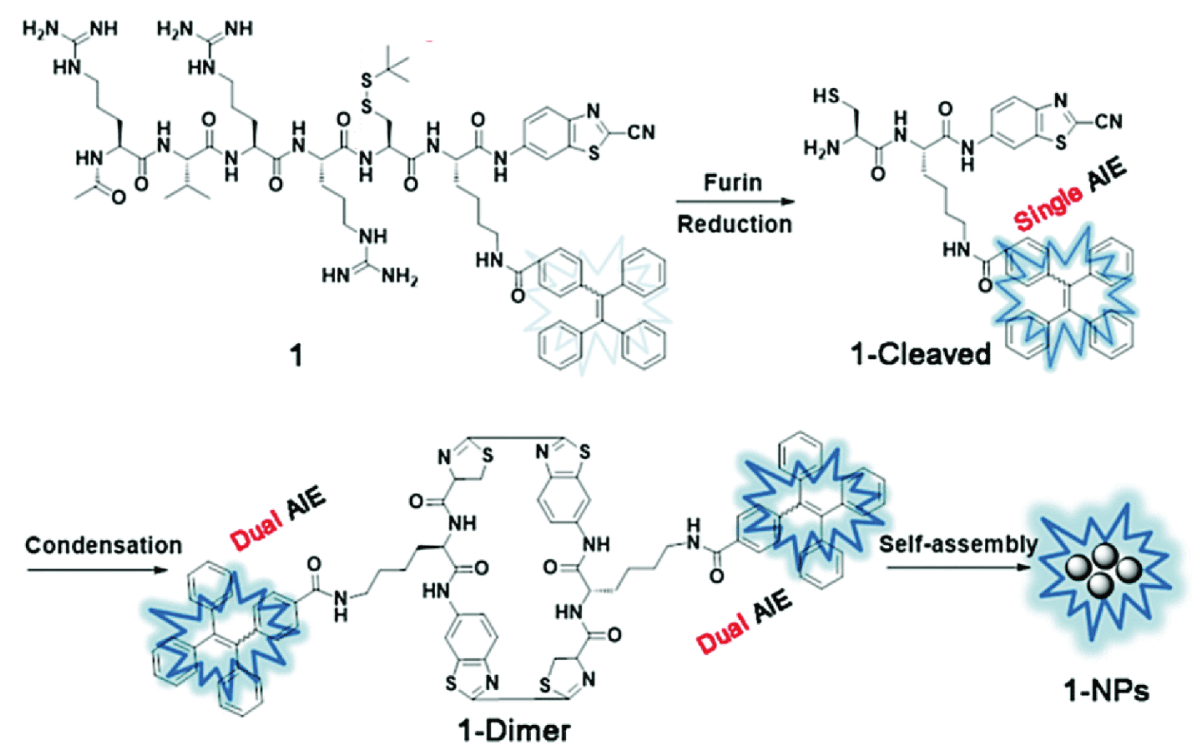

Figure 14 The detection mechanism for sensing furin activity based on AIE dual aggregation effect. Reproduced with permission from Ref. [71]. Copyright 2017, the Royal Society of Chemistry.

low as well as almost no specific interaction toward normal tissues. Firstly, an azide-modified acetyl sialic acid (AzAcSA) is modified on the cell membrane by a metabolic engineering. The BCN-TPET-TEG incubated with $4 \mathrm{~T} 1$ cells then undergo bio-orthogonal reaction. The fluorescence of $4 \mathrm{~T} 1$ cells treated with AzAcSA gradually increase over time. Furthermore, the fluorescence on the 4T1 cells coincides with the fluorescence of the cell membrane fluorescence tracker indicating that BCNTPET-TEG is labeled on the cell membrane by a click reaction. By using 2',7'-dichlorofluorescein diacetate as a probe for detecting singlet oxygen, AzAcSA and BCNTPET-TEG treated 4T1 cells show significant green fluorescence after illumination, indicating bio-orthogonally labeled BCN-TPET-TEG is capable of producing singlet oxygen. The ability of the probe for the generation of singlet oxygen can be used for imaging guided photodynamic therapy.

Liu's group also developed some bio-orthogonal lightup probes, such as TPETSAl [68] and TPBAl [69], which can both light their fluorescence up on the basis of a click reaction with azide moieties on membranes of the cancer cells, fulfilling imaging and ablation of the specific cancer cells. And Liu et al. [70] further reported a novel assay to realize in vivo bacterial metabolic labeling and precise antibacterial therapy.

Heterocycle

AIE effects are widely used for the detection of biomo- lecules. However, the "smart" strategy to develop AIE fluorophore aggregates as well as additional enhancement of the fluorescence is still a challenge. Liang et al. [71] achieved the dual aggregation of AIE by combining the tetraphenylethylene with AIE effect and the aggregation of furin in response to 2-cyanobenzothiazole(CBT)-Cys. Therefore, the fluorescence imaging and detection of furin can be achieved by using the AIE dual aggregation effect. To achieve this goal, they designed and synthesized Ac-Arg-Val-Arg-Arg-Cys(StBu)-Lys(TPE)-CBT (probe 1). The peptide sequence Ac-Arg-Val-Arg-Arg (RVRR) not only improves the water solubility of the probe but also is a substrate for the furin. The CBT group and the Cys containing a disulfide bond are capable of forming CBT-Cys aggregates while the TPE is attached to the side chain of lysine. As shown in Fig. 14, when the probe gets into the cell with overexpression of furin, such as MDAMB-468 cells, the intracellular GSH reduces the disulfide bond in cysteine. Meanwhile, the RVRR peptide substrate is treated with furin. The probe is hydrolyzed to produce an active 1-Cleaved. Theoretically the 1-Cleaved is more hydrophobic than 1 resulting in the first aggregation of TPE. The 1,2-aminothiol in Cys reacts with the CN group on the 1-Cleaved CBT group to form a more hydrophobic dimer. The dimer further assembles or aggregates in the region of the furin activation. The conversion from 1Cleaved to the 1-dimer as well as the 1-NPs further facilitates the AIE luminophore, thereby further enhancing the fluorescence of the AIE. Through a dual fluorescence 
enhancement strategy, the probe becomes more sensitive for the detection of the activity of furin in tumor cells. To confirm the above hypothesis, a probe of Ac-Arg-Lys (TPE)-Arg-Cys(StBu)-Arg-Val-CBT(1-Scr) was synthesized which did not respond to furin as a control. In summary, the 1-Cleaved has only a single AIE effect but it is an active intermediate and therefore difficult to capture.

\section{CONCLUSIONS AND PERSPECTIVES}

In this review, we discussed the fluorescent probes with AIE feature on the basis of bond cleavage and formation strategy. In particular, the design strategy of fluorescent probe with AIE characteristics was explained. The fluorescent probes were divided into two classes on the basis of bond cleavage as well as formation. The bond cleavage included amido bond, ester bond, boron ester bond, phosphodiester bond, disulfide bond, $\mathrm{C}-\mathrm{O}$ bond and $\mathrm{S}-\mathrm{O}$ bond; the bond formation contained $\mathrm{C}-\mathrm{C}$ bond, boron ester bond, thioester bond, nitrogen heterocycle bond as well as heterocycle bond. Although significant progress has been made in the development of fluorescent probes with AIE feature on the basis of bond cleavage as well as formation strategy, challenges still remain in the development of these probes. As most of AIEgens emit the fluorescence in the ultraviolet-visible light range, thus the fluorescent probes with AIE feature in near infrared region based on the change of covalent bond are expected to develop. In addition, the determination of multiple analytes by one probe is desirable.

Received 26 January 2019; accepted 22 February 2019; published online 25 March 2019

1 Zhuang Y, Shang C, Lou X, et al. Construction of AIEgens-based bioprobe with two fluorescent signals for enhanced monitor of extracellular and intracellular telomerase activity. Anal Chem, 2017, 89: 2073-2079

2 Zhuang Y, Zhang M, Chen B, et al. Quencher group induced high specificity detection of telomerase in clear and bloody urines by AIEgens. Anal Chem, 2015, 87: 9487-9493

3 Cheng Y, Sun C, Ou X, et al. Dual-targeted peptide-conjugated multifunctional fluorescent probe with AIEgen for efficient nucleus-specific imaging and long-term tracing of cancer cells. Chem Sci, 2017, 8: 4571-4578

4 Lou X, Zhuang Y, Zuo X, et al. Real-time, quantitative lighting-up detection of telomerase in urines of bladder cancer patients by AIEgens. Anal Chem, 2015, 87: 6822-6827

5 Zhuang Y, Huang F, Xu Q, et al. Facile, fast-responsive, and photostable imaging of telomerase activity in living cells with a fluorescence turn-on manner. Anal Chem, 2016, 88: 3289-3294

6 Zhuang Y, Xu Q, Huang F, et al. Ratiometric fluorescent bioprobe for highly reproducible detection of telomerase in bloody urines of bladder cancer patients. ACS Sens, 2016, 1: 572-578

7 Long Z, Zhan S, Gao P, et al. Recent advances in solid nanopore/ channel analysis. Anal Chem, 2018, 90: 577-588

8 Zhang X, Lou X, Xia F. Advances in the detection of telomerase activity using isothermal amplification. Theranostics, 2017, 7: $1847-1862$

9 Wang $\mathrm{X}, \mathrm{Xu} \mathrm{M}$, Huang $\mathrm{K}$, et al. AIEgens/nucleic acid nanostructures for bioanalytical applications. Chem Asian J, 2019, 14: 689-699

10 Jia Y, Gao P, Zhuang Y, et al. Facile probe design: Fluorescent amphiphilic nucleic acid probes without quencher providing telomerase activity imaging inside living cells. Anal Chem, 2016, 88: $6621-6626$

11 Yi X, Dai J, Han Y, et al. A high therapeutic efficacy of polymeric prodrug nano-assembly for a combination of photodynamic therapy and chemotherapy. Commun Biol, 2018, 1: 202

12 Li X, Zhu S, Xu B, et al. Self-assembled graphene quantum dots induced by cytochrome c: A novel biosensor for trypsin with remarkable fluorescence enhancement. Nanoscale, 2013, 5: 77767779

13 Lu H, Xu B, Dong Y, et al. Novel fluorescent $\mathrm{pH}$ sensors and a biological probe based on anthracene derivatives with aggregationinduced emission characteristics. Langmuir, 2010, 26: 6838-6844

$14 \mathrm{Li} \mathrm{X}, \mathrm{Ma} \mathrm{K}, \mathrm{Lu} \mathrm{H}$, et al. Highly sensitive determination of ssDNA and real-time sensing of nuclease activity and inhibition based on the controlled self-assembly of a 9,10-distyrylanthracene probe. Anal Bioanal Chem, 2014, 406: 851-858

15 Gao H, Zhang X, Chen C, et al. Unity makes strength: How aggregation-induced emission luminogens advance the biomedical field. Adv Biosys, 2018, 2: 1800074

$16 \mathrm{Ni}$ X, Zhang X, Duan X, et al. Near-infrared afterglow luminescent aggregation-induced emission dots with ultrahigh tumor-to-liver signal ratio for promoted image-guided cancer surgery. Nano Lett, 2019, 19: 318-330

17 Qi J, Chen C, Ding D, et al. Aggregation-induced emission luminogens: Union is strength, gathering illuminates healthcare. Adv Healthcare Mater, 2018, 7: 1800477

18 Zhao Z, Chen C, Wu W, et al. Highly efficient photothermal nanoagent achieved by harvesting energy via excited-state intramolecular motion within nanoparticles. Nat Commun, 2019, 10: 768

19 Cheng Y, Sun C, Liu R, et al. A multifunctional peptide-conjugated AIEgen for efficient and sequential targeted gene delivery into the nucleus. Angew Chem Int Ed, 2019

20 Ma K, Zhang F, Sayyadi N, et al. "Turn-on" fluorescent aptasensor based on AIEgen labeling for the localization of IFN- $\gamma$ in live cells. ACS Sens, 2018, 3: 320-326

21 Liu L, Zhang F, Xu B, et al. Silica nanoparticles based on an AIEactive molecule for ratiometric detection of RNS in vitro. J Mater Chem B, 2017, 5: 9197-9203

22 Li X, Ma K, Zhu S, et al. Fluorescent aptasensor based on aggregation-induced emission probe and graphene oxide. Anal Chem, 2014, 86: 298-303

23 Ma K, Wang $\mathrm{H}$, Li H, et al. A label-free aptasensor for turn-on fluorescent detection of ATP based on AIE-active probe and watersoluble carbon nanotubes. Sensor Actuat B-Chem, 2016, 230: 556558

24 Ma K, Wang H, Li H, et al. Label-free detection for SNP using AIE probes and carbon nanotubes. Sensor Actuat B-Chem, 2017, 253: 92-96 
25 Ma K, Wang $\mathrm{H}$, Li X, et al. Turn-on sensing for $\mathrm{Ag}^{+}$based on AIEactive fluorescent probe and cytosine-rich DNA. Anal Bioanal Chem, 2015, 407: 2625-2630

26 Wang H, Ma K, Xu B, et al. Tunable supramolecular interactions of aggregation-induced emission probe and graphene oxide with biomolecules: An approach toward ultrasensitive label-free and "turn-on" DNA sensing. Small, 2016, 12: 6613-6622

27 Zhang S, Ma L, Ma K, et al. Label-free aptamer-based biosensor for specific detection of chloramphenicol using AIE probe and graphene oxide. ACS Omega, 2018, 3: 12886-12892

28 Yuan Y, Zhang CJ, Kwok RTK, et al. Light-up probe based on AIEgens: Dual signal turn-on for caspase cascade activation monitoring. Chem Sci, 2017, 8: 2723-2728

29 Cheng Y, Huang F, Min X, et al. Protease-responsive prodrug with aggregation-induced emission probe for controlled drug delivery and drug release tracking in living cells. Anal Chem, 2016, 88: 8913-8919

30 Yuan Y, Zhang CJ, Gao M, et al. Specific light-up bioprobe with aggregation-induced emission and activatable photoactivity for the targeted and image-guided photodynamic ablation of cancer cells. Angew Chem Int Ed, 2015, 54: 1780-1786

31 Li J, Lee WY, Wu T, et al. Detection of matrix metallopeptidase 13 for monitoring stem cell differentiation and early diagnosis of osteoarthritis by fluorescent light-up probes with aggregation-induced emission characteristics. Adv Biosys, 2018, 2: 1800010

32 Wang Y, Wu X, Cheng Y, et al. A fluorescent switchable AIE probe for selective imaging of dipeptidyl peptidase-4 in vitro and in vivo and its application in screening dpp-4 inhibitors. Chem Commun, 2016, 52: 3478-3481

33 Hou X, Zeng F, Wu S. A fluorescent assay for $\gamma$-glutamyltranspeptidase via aggregation induced emission and its applications in real samples. Biosens Bioelectron, 2016, 85: 317-323

34 Zhang R, Zhang CJ, Feng G, et al. Specific light-up probe with aggregation-induced emission for facile detection of chymase. Anal Chem, 2016, 88: 9111-9117

35 Wang $\mathrm{H}$, Huang $\mathrm{Y}$, Zhao $\mathrm{X}$, et al. A novel aggregation-induced emission based fluorescent probe for an angiotensin converting enzyme (ACE) assay and inhibitor screening. Chem Commun, 2014, 50: 15075-15078

36 Han A, Wang H, Kwok RTK, et al. Peptide-induced AIEgen selfassembly: A new strategy to realize highly sensitive fluorescent light-up probes. Anal Chem, 2016, 88: 3872-3878

37 Kim TI, Jin H, Bae J, et al. Excimer emission-based fluorescent probe targeting caspase-3. Anal Chem, 2017, 89: 10565-10569

38 Liang J, Shi H, Kwok RTK, et al. Distinct optical and kinetic responses from $\mathrm{e} / \mathrm{z}$ isomers of caspase probes with aggregation-induced emission characteristics. J Mater Chem B, 2014, 2: 43634370

39 Yuan Y, Zhang R, Cheng X, et al. A FRET probe with AIEgen as the energy quencher: Dual signal turn-on for self-validated caspase detection. Chem Sci, 2016, 7: 4245-4250

40 Ding D, Liang J, Shi H, et al. Light-up bioprobe with aggregationinduced emission characteristics for real-time apoptosis imaging in target cancer cells. J Mater Chem B, 2014, 2: 231-238

41 Han $\mathrm{H}$, Teng $\mathrm{W}$, Chen $\mathrm{T}$, et al. A cascade enzymatic reaction activatable gemcitabine prodrug with an AIE-based intracellular light-up apoptotic probe for in situ self-therapeutic monitoring. Chem Commun, 2017, 53: 9214-9217

42 Shi H, Kwok RTK, Liu J, et al. Real-time monitoring of cell apoptosis and drug screening using fluorescent light-up probe with aggregation-induced emission characteristics. J Am Chem Soc, 2012, 134: 17972-17981

43 Shi H, Zhao N, Ding D, et al. Fluorescent light-up probe with aggregation-induced emission characteristics for in vivo imaging of cell apoptosis. Org Biomol Chem, 2013, 11: 7289-7296

44 Yuan Y, Zhang CJ, Kwok RTK, et al. Light-up probe for targeted and activatable photodynamic therapy with real-time in situ reporting of sensitizer activation and therapeutic responses. Adv Funct Mater, 2015, 25: 6586-6595

45 Li J, Leung CWT, Wong DSH, et al. Photocontrolled siRNA delivery and biomarker-triggered luminogens of aggregation-induced emission by up-conversion $\mathrm{NaYF}_{4}: \mathrm{Yb}^{3+} \mathrm{Tm}^{3+} @ \mathrm{SiO}_{2}$ nanoparticles for inducing and monitoring stem-cell differentiation. ACS Appl Mater Interfaces, 2017, acsami.7b00845

46 Yuan Y, Kwok RTK, Tang BZ, et al. Targeted theranostic platinum (IV) prodrug with a built-in aggregation-induced emission lightup apoptosis sensor for noninvasive early evaluation of its therapeutic responses in situ. J Am Chem Soc, 2014, 136: 2546-2554

47 Shi J, Deng Q, Wan C, et al. Fluorometric probing of the lipase level as acute pancreatitis biomarkers based on interfacially controlled aggregation-induced emission (AIE). Chem Sci, 2017, 8: 6188-6195

48 Shi J, Zhang S, Zheng M, et al. A novel fluorometric turn-on assay for lipase activity based on an aggregation-induced emission (AIE) luminogen. Sensor Actuat B-Chem, 2017, 238: 765-771

49 Yuan Y, Zhang CJ, Xu S, et al. A self-reporting AIE probe with a built-in singlet oxygen sensor for targeted photodynamic ablation of cancer cells. Chem Sci, 2016, 7: 1862-1866

50 Liu GJ, Long Z, Lv HJ, et al. A dialdehyde-diboronate-functionalized AIE luminogen: Design, synthesis and application in the detection of hydrogen peroxide. Chem Commun, 2016, 52: 1023310236

51 Song Z, Mao D, Sung SHP, et al. Activatable fluorescent nanoprobe with aggregation-induced emission characteristics for selective in vivo imaging of elevated peroxynitrite generation. Adv Mater, 2016, 28: 7249-7256

52 Min X, Zhuang Y, Zhang Z, et al. Lab in a tube: Sensitive detection of micrornas in urine samples from bladder cancer patients using a single-label DNA probe with AIEgens. ACS Appl Mater Interfaces, 2015, 7: 16813-16818

53 Min X, Zhang M, Huang F, et al. Live cell microrna imaging using exonuclease III-aided recycling amplification based on aggregation-induced emission luminogens. ACS Appl Mater Interfaces, 2016, 8: 8998-9003

54 Wang X, Dai J, Min X, et al. DNA-conjugated amphiphilic aggregation-induced emission probe for cancer tissue imaging and prognosis analysis. Anal Chem, 2018, 90: 8162-8169

55 Min X, Xia L, Zhuang Y, et al. An AIEgens and exonuclease iii aided quadratic amplification assay for detecting and cellular imaging of telomerase activity. Sci Bull, 2017, 62: 997-1003

56 Ou X, Lou X, Xia F. A highly sensitive DNA-AIEgen-based "turnon" fluorescence chemosensor for amplification analysis of $\mathrm{Hg}^{2+}$ ions in real samples and living cells. Sci China Chem, 2017, 60: 663-669

57 Shi J, Deng Q, Li Y, et al. A rapid and ultrasensitive tetraphenylethylene-based probe with aggregation-induced emission for direct detection of $\alpha$-amylase in human body fluids. Anal Chem, 2018, 90: 13775-13782

58 Zhang $\mathrm{P}$, Nie X, Gao M, et al. A highly selective fluorescent nanoprobe based on AIE and ESIPT for imaging hydrogen sulfide in 
live cells and zebrafish. Mater Chem Front, 2017, 1: 838-845

59 Yuan Y, Xu S, Zhang CJ, et al. Dual-targeted activatable photosensitizers with aggregation-induced emission (AIE) characteristics for image-guided photodynamic cancer cell ablation. J Mater Chem B, 2016, 4: 169-176

60 Yuan Y, Kwok RTK, Feng G, et al. Rational design of fluorescent light-up probes based on an AIE luminogen for targeted intracellular thiol imaging. Chem Commun, 2014, 50: 295-297

61 Cheng Y, Dai J, Sun C, et al. An intracellular $\mathrm{H}_{2} \mathrm{O}_{2}$-responsive AIEgen for the peroxidase-mediated selective imaging and inhibition of inflammatory cells. Angew Chem Int Ed, 2018, 57: 3123-3127

62 Wang X, Hu J, Zhang G, et al. Highly selective fluorogenic multianalyte biosensors constructed via enzyme-catalyzed coupling and aggregation-induced emission. J Am Chem Soc, 2014, 136: 9890-9893

63 Qi J, Chen C, Zhang X, et al. Light-driven transformable optical agent with adaptive functions for boosting cancer surgery outcomes. Nat Commun, 2018, 9: 1848

$64 \mathrm{Xu} \mathrm{X}$, Zhao W, Gao P, et al. Coordination of the electrical and optical signals revealing nanochannels with an 'onion-like' gating mechanism and its sensing application. NPG Asia Mater, 2016, 8: e234

65 Liu Y, Deng C, Tang L, et al. Specific detection of D-glucose by a tetraphenylethene-based fluorescent sensor. J Am Chem Soc, 2011, 133: $660-663$

66 Chang J, Li H, Hou T, et al. Paper-based fluorescent sensor for rapid naked-eye detection of acetylcholinesterase activity and organophosphorus pesticides with high sensitivity and selectivity. Biosens Bioelectron, 2016, 86: 971-977

67 Hu F, Mao D, Kenry D, et al. A light-up probe with aggregationinduced emission for real-time bio-orthogonal tumor labeling and image-guided photodynamic therapy. Angew Chem Int Ed, 2018, 57: 10182-10186

68 Yuan Y, Xu S, Cheng X, et al. Bioorthogonal turn-on probe based on aggregation-induced emission characteristics for cancer cell imaging and ablation. Angew Chem Int Ed, 2016, 55: 6457-6461

69 Hu F, Yuan Y, Wu W, et al. Dual-responsive metabolic precursor and light-up AIEgen for cancer cell bio-orthogonal labeling and precise ablation. Anal Chem, 2018, 90: 6718-6724

70 Mao D, Hu F, Kenry F, et al. Metal-organic-framework-assisted in vivo bacterial metabolic labeling and precise antibacterial therapy. Adv Mater, 2018, 30: 1706831

71 Liu X, Liang G. Dual aggregation-induced emission for enhanced fluorescence sensing of furin activity in vitro and in living cells. Chem Commun, 2017, 53: 1037-1040

Acknowledgements This work was supported by the National Natural Science Foundation of China (21525523, 21722507, 21574048 and 21874121), the National Basic Research Program of China (973 Program, 2015CB932600), the National Key R\&D Program of China (2017YFA0208000 and 2016YFF0100800), and the Natural Science Foundation of Zhejiang Province of China (LY18B050002).

Author contributions Lou $\mathrm{X}$ and $\mathrm{Xia} F$ designed the topic and framework of this review; Wang X, Xu M, Wang Q and Hu Q collected the data; Xu M wrote the review with the help of Wang X. All authors contributed to the general discussion.
Conflict of interest The authors declare no conflict of interest.

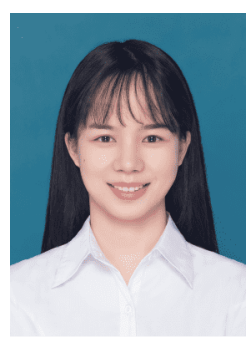

Min Xu obtained her BSc degree from China University of Geosciences in 2017, and she is currently studying for her Master's degree at China University of Geosciences under the supervision of Prof. Xiaoding Lou and Prof. Fan Xia. Her current research is focused on the fluorescent nanoprobes.

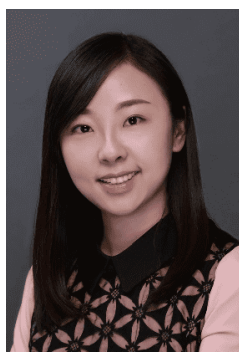

Xiaoding Lou received her BSc degree from Hubei Engineering University (China) in 2007. She studied organic chemistry at Wuhan University (China), where she received her $\mathrm{PhD}$ degree in 2012 under the supervision of Prof. Zhen Li. She then worked for one year as a research associate in Prof. Ben Zhong Tang's group at Hong Kong University of Science and Technology. In 2013, she became an assistant professor and associate professor at Huazhong University of Science and Technology (China), respectively. In 2016, she worked at the University of California, Santa Barbara in Prof. Alan J. Heeger's group. Since 2017, she has been a professor of analytical chemistry at China University of Geosciences (Wuhan). Her scientific interest is focused on the chemical and biosensor field.

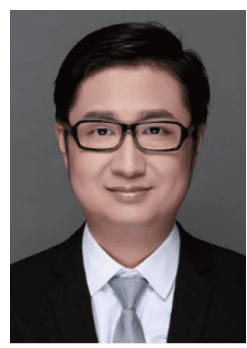

Fan Xia received his BSc degree from Huazhong University of Science and Technology (HUST) in China in 2003. He studied physical chemistry at the Institute of Chemistry, Chinese Academy of Sciences (ICCAS), where he received his $\mathrm{PhD}$ degree in 2008 under the supervision of Prof. Lei Jiang. He then worked as a postdoctoral fellow in Prof. Alan J. Heeger's group at the University of California, Santa Barbara. He joined HUST as part of the 1000 Young Talents Program in 2012 and became professor at HUST. He is currently a professor and Dean of Faculty of Materials Science and Chemistry, China University of Geosciences (Wuhan). His scientific interest is focused on bioanalytical chemistry.

\section{基于共价键变化的靶标响应性AIE苂光探针 \\ 许敏 ${ }^{1}$, 汪旭东 ${ }^{2}$, 王全 ${ }^{1}$, 胡钦铂 ${ }^{1}$, 黄开勋 ${ }^{2}$, 娄簕叮 ${ }^{1 *}$, 夏帆 ${ }^{1,2^{*}}$}

摘要 苂光探针是检测生物或者环境中相关物质的重要手段. 传统 的苂光探针由于聚集诱导猝灭引起的选择性差异和容易光漂白等 问题限制了其应用. 此外, 在传统苂光探针的设计中通常引入猝灭 基团, 从而增加了探针合成及分离的难度. 一类新型的具有聚集诱 导发光(AIE)特征的苂光染料解决了上述问题. 利用AIE发光团的 独特性质, 构建发光团与识别基团偶联的特异性“关开型”探针, 这 些探针具有背景低、选择性好和光稳定性强的优点. 本综述主要 概括并讨论了通过共价键断裂以及新键形成策略开发的具有AIE 特征的荧光探针, 并对其进行了展望. 